Audiology

Neurotology
Audiol Neurotol 2015;20:39-50

DOI: $10.1159 / 000362780$
Received: October 7, 2013

Accepted after revision: April 9, 2014 Published online: December 9, 2014

\title{
Quantifying the Vestibulo-Ocular Reflex with Video-Oculography: Nature and Frequency of Artifacts
}

\author{
Georgios Mantokoudis $^{a} \quad$ Ali S. Saber Tehrania Jorge C. Kattah ${ }^{c}$ \\ Karin Eibenberger $^{b}$ Cynthia I. Guede ${ }^{c}$ David S. Zee ${ }^{a}$ David E. Newman-Toker ${ }^{a, b}$ \\ Departments of a Neurology and ${ }^{b}$ Otolaryngology - Head and Neck Surgery, Johns Hopkins University School of \\ Medicine, Baltimore, Md., and 'University of Illinois College of Medicine, Peoria, III., USA
}

\section{Key Words}

Eye movement measurements · Artifacts · Vertigo ·

Vestibular neuritis · Stroke

\begin{abstract}
Video-oculography devices are now used to quantify the vestibulo-ocular reflex (VOR) at the bedside using the head impulse test (HIT). Little is known about the impact of disruptive phenomena (e.g. corrective saccades, nystagmus, fixation losses, eye-blink artifacts) on quantitative VOR assessment in acute vertigo. This study systematically characterized the frequency, nature, and impact of artifacts on HIT VOR measures. From a prospective study of 26 patients with acute vestibular syndrome (16 vestibular neuritis, 10 stroke), we classified findings using a structured coding manual. Of 1,358 individual HIT traces, $72 \%$ had abnormal disruptive saccades, $44 \%$ had at least one artifact, and $42 \%$ were uninterpretable. Physicians using quantitative recording devices to measure head impulse VOR responses for clinical diagnosis should be aware of the potential impact of disruptive eye movements and measurement artifacts.
\end{abstract}

(c) 2014 S. Karger AG, Basel
(C) 2014 S. Karger AG, Basel

$1420-3030 / 14 / 0201-0039 \$ 39.50 / 0$

\section{Introduction}

The head impulse test (HIT), first described by Halmagyi and Curthoys in 1988 [Halmagyi and Curthoys, 1988], is a critical component of bedside assessment of vestibular function. The technique leverages a high-acceleration $\left(1,000-6,000^{\circ} / \mathrm{s}^{2}\right)$, rapid $\left(100-200^{\circ} / \mathrm{s}\right)$, low-amplitude $\left(10-20^{\circ}\right)$ head rotation to assess the integrity of the vestibulo-ocular reflex (VOR). The patient visually fixates on a stationary target. If the clinician detects a corrective eye movement immediately after the head rotation ('refixation saccade'), a deficient VOR response is indirectly inferred. The HIT can be used to assess the function of each semicircular canal individually [Halmagyi et al., 2001].

In specialty practice, the horizontal HIT (h-HIT) is now widely used to assist in clinical diagnosis of peripheral vestibular disorders [Curthoys, 2012; Strupp and Brandt, 2013] such as vestibular neuritis [Blödow et al., 2013], unilateral vestibular loss [Weber et al., 2008], and bilateral vestibulopathy [Petersen et al., 2013]. Impulsive testing of the vertical semicircular canals is useful for diagnosing inferior vestibular neuritis [Kim and Kim, 2012], postsurgical follow-up of superior canal dehis-

\section{KARGER 125}

E-Mail karger@karger.com www.karger.com/aud
David E. Newman-Toker, MD, PhD, Associate Professor

Department of Neurology, The Johns Hopkins University School of Medicine The Johns Hopkins Hospital, Meyer Building 8-154, 600 North Wolfe Street Baltimore, MD 21287 (USA)

E-Mail toker@jhu.edu 
cence syndrome repair [Janky et al., 2012], or to assess the success of vestibular neurectomy [Lehnen et al., 2004].

In the emergency department, the h-HIT is also the single most sensitive test for the detection of posterior fossa strokes in patients with acute, continuous vertigo presentations [Newman-Toker et al., 2008; Kattah et al., 2009; Tarnutzer et al., 2011; Newman-Toker et al., 2013a; Newman-Toker et al., 2013b]. In these patients, experts qualitatively assessing three bedside oculomotor tests known as 'H.I.N.T.S.' (Head Impulse, Nystagmus, Test of Skew) can diagnose stroke more accurately at the bedside than early MRI of the brain [Kattah et al., 2009; NewmanToker et al., 2013a].

A noninvasive video-oculography (VOG) device is now available that measures the HIT VOR directly (i.e. without relying on the refixation saccade) and objectively [MacDougall et al., 2009]. Its measurement accuracy has been validated [MacDougall et al., 2009; Weber et al., 2009; Macdougall et al., 2013] against the research laboratory gold standard for oculomotor recordings (magnetic scleral search coils [Robinson, 1963]). The device was recently approved by the US Food and Drug Administration as safe and effective for vestibular testing [GN Otometrics]. This VOG device, sometimes referred to as a 'video HIT' device, consists of a pair of lightweight goggles, similar in appearance to swimming goggles, with an embedded high-speed ( $\geq 250$ frames/s) infrared video camera to track eye movements and inertial accelerometer in the frame to track head movements [GN Otometrics, 2013b].

The VOG HIT approach ensures accurate assessment of VOR function, including avoiding the pitfall of being fooled by 'covert saccades' (refixation saccades occurring during the HIT head rotation, making them invisible even to experts when performed clinically at the bedside without quantification) [Weber et al., 2008]. Quantification offers the added benefit of monitoring progression or recovery through serial testing and follow-up. The impulse device has already been used in the outpatient setting to help diagnose and monitor peripheral vestibular disorders [MacDougall et al., 2009; Weber et al., 2009; Manzari et al., 2011; Manzari et al., 2013]. Since VOG HIT provides complementary information to routine caloric testing of vestibular function [Park et al., 2005], it is likely to become a routine component of vestibular function test batteries in otolaryngology practice [Curthoys, 2012].

The VOG device also offers the potential of broad dissemination of these approaches to frontline healthcare settings by assisting nonspecialists (e.g. emergency physicians) with HIT testing and interpretation, which is crucial for accurate diagnosis of patients with acute dizziness or vertigo [Kattah et al., 2009; Tarnutzer et al., 2011; Newman-Toker, 2012; Newman-Toker et al., 2013a]. We recently showed that the device can accurately discriminate central (stroke) from peripheral (vestibular neuritis) causes of the acute vestibular syndrome in the emergency department [Newman-Toker et al., 2013b].

Despite major advances in eye movement recording technology from the 1970s [Young and Sheena, 1975] to the present [Eggert, 2007], however, VOG recordings remain imperfect. VOG HIT results are subject to measurement error for three main reasons: (1) it is an inherently 'noisier' technique than scleral search coil recordings because of goggle slippage, imperfect pupil-tracking algorithms, and lower sampling rates, (2) application to acute vestibular patients with intrusive eye movement disorders that affect normal eye position and tracking (e.g. spontaneous nystagmus in acute vestibular neuritis), and (3) use in less-well controlled clinical settings (e.g. emergency department) where environmental conditions (e.g. patient position or room lighting) may vary across trials or patients. As a result, expert assessment and interpretation of HIT results is still required.

Thus far, there has been little study of artifacts in quantitative VOG HIT testing, including how they affect VOR measurement accuracy or clinical interpretation. We sought to characterize the nature of artifacts in VOG HIT recordings obtained from patients with acute vertigo in the emergency department. To accomplish this, we first developed a structured coding manual of known artifacts. In this article, we describe frequency and types of artifacts in patients with both normal and abnormal VOR. In a separate article, we will further detail the impact of these artifacts on the reliability of gain ratio measurements.

\section{Material and Methods}

\section{Study Population}

In this prospective cross-sectional study, we enrolled patients with acute vestibular syndrome between August 2011 and December 2012 from the emergency departments of two academic tertiary referral centers (OSF Saint Francis Medical Center, Peoria, Ill., USA, and Johns Hopkins Hospital, Baltimore, Md., USA). Patients visiting the emergency department during recruitment hours (convenience sampling) were systematically screened for acute vestibular syndrome, defined as $>24$ h of acute continuous dizziness/vertigo, nausea/vomiting, head motion intolerance, gait unsteadiness, and nystagmus. Patients with lim-
40

Audiol Neurotol 2015;20:39-50 DOI: $10.1159 / 000362780$
Mantokoudis et al. 
ited vision or known prior vestibulopathy or oculomotor disorder were excluded.

We included here all acute vestibular syndrome patients with a definitive diagnosis for the cause of dizziness-stroke, or vestibular neuritis/labyrinthitis. Strokes were all confirmed by MRI brain with diffusion-weighted imaging in the first 10 days after symptom onset. Vestibular neuritis/labyrinthitis was diagnosed based on (1) appropriate clinical history and exam features [including either unilateral caloric weakness $(>25 \%)$ or abnormal HIT (low gain $<0.68$ or gain asymmetry $>20 \%$ ), no skew deviation, unilateral direction of nystagmus beating opposite the VOR deficit], (2) negative MRI-diffusion-weighted imaging (i.e. no acute stroke), and (3) 3 months of follow-up without evidence of subsequent posteriorfossa stroke based on case history and exam findings. Enrolled patients who did not undergo MRI brain for clinical care purposes (i.e. those without a definitive final diagnosis) were not included. The study was approved by the institutional review boards of both institutions. All patients provided written informed consent. VOG diagnostic results (but not artifact analysis) from a subset of these patients $(n=12)$ have been presented in a prior publication [Newman-Toker et al., 2013b].

\section{h-HIT VOG Recordings}

Patients underwent a structured bedside neuro-otologic exam, generally in the emergency department [Johns Hopkins, by trained research fellows (G.M. or A.S.S.T.)] or vestibular clinic [OSF Saint Francis, by a trained nurse (C.I.G.)]. The three examiners had similar training and experience, and their accuracy in performing $\mathrm{h}$ HIT VOR measures was monitored for consistency by assessing the rate of failed HIT maneuvers (i.e. those automatically rejected by the device software). We captured h-HIT measurements using a lightweight, portable VOG device (ICS Impulse; GN Otometrics, Taastrup, Denmark). Patients were either seated in bed or in a chair, and were asked to fix their gaze on a distant target $(>1.5 \mathrm{~m})$ on the opposite wall or dividing curtain of a room in the emergency department. Eye position was calibrated using laser targets projected forward from the goggles. After calibration, the examiners performed a series of inward HITs (i.e. centripetal, lateral-tocenter head rotations) toward each ear with the examiner positioned either in front of the patient, placing his/her hand on the patient's jaw [Halmagyi and Curthoys, 1988] (Johns Hopkins), or behind the patient, holding the patient's head on the top [Weber et al., 2008] (OSF Saint Francis). At the Johns Hopkins site, we examined from the front because many emergency department exam beds do not readily permit the examiner to be located behind the patient; to avoid obscuring the patient's line of sight, the examiner was displaced slightly to the right or left (generally at the side of the patient's bed), and the patient looked around or over the shoulder of the examiner at the fixation target on the far wall or curtain.

Target head velocity was between 100 and $200 \%$ s and head displacement ranged between 5 and $20^{\circ}$. The target number of HITs was prespecified to a range (10-50) instead of a number because (1) not all correctly performed HITs captured by the device are accepted during the final automated processing step (e.g. rejected for eye tracking loss), (2) prior to the study, we did not know the optimal number of HITs to perform, and (3) prior to the study, we did not know the number of HITs that would be tolerated by acute vestibular patients. Thus, after 10 HITs we continued testing within the range as tolerated. If spontaneous nystagmus was present, device software was operated in the nystagmus-adjusted interpre- tation mode, which alters filtering algorithms for determining inadequate impulses and calculates compensated VOR gain measures by accounting for the spontaneous, slow-phase drift of the eye.

\section{Data Processing}

All h-HITs collected and accepted by the algorithm of the device software were stored and assessed for artifacts by a single, masked trained rater (G.M.) 'offline' by processing raw quantitative data exported from the ICS Impulse device using Matlab R2012b (Mathworks, Natick, Mass., USA) (online suppl. Appen$\operatorname{dix} \mathrm{A}$; for all online suppl. material, see www.karger.com/ doi/10.1159/000362780). A second, independent rater (A.S.S.T.) recoded a random $10 \%$ subsample to assess interrater reliability. Each individual head and eye velocity trace (i.e. a single HIT maneuver) was deidentified and displayed in random order using a specially designed Matlab script. This allowed raters to remain masked to the patient's results and diagnosis. The random-order presentation also served to avoid the problem of order effects in coding - specifically being influenced by a pattern emerging from batch analysis of HIT results from the same patient. This decontextualized interpretation approach was conservative with respect to determining a correct, interpretable trace without artifacts (i.e. increased the chances of counting a trace as an artifact, creating a high standard for an acceptable HIT).

\section{Outcome Measures}

We created a coding manual (online suppl. Appendix B) for the classification of HIT results based on physiologic trace morphology and associated corrective saccades (fig. 1). Raters assessed three distinct classes of eye movement findings in each HIT physiologic trace: (1) slow-phase VOR, (2) fast-phase eye movements (i.e. saccades), and (3) artifacts (online suppl. Appendix B). Figure 2 shows exemplar research subject traces demonstrating typical normal and abnormal physiologic responses, without significant artifacts. Figure 3 shows exemplar traces from a normal subject demonstrating different types of deliberately induced artifacts. Note that artifacts shown in figure 3 were created and reliably reproduced under laboratory conditions on healthy subjects, so their origins are definitively known. Artifacts assessed in HIT tracings from patients with acute vestibular syndrome were identified post hoc based on morphologic similarity to these known artifacts, without specific foreknowledge of their true cause.

We coded eight different types of artifacts (fig. 3): phase shift, inappropriately high gain, pseudo-saccades, multiple VOR peaks (i.e. non-bell-shaped curve), excessive post-HIT bounce, eye moves opposite the expected slow phase VOR direction (i.e. with rather than in opposition to the head), trace oscillations (noisy baseline), and unclassifiable artifacts (i.e. multiple different artifact morphologies or unrecognizable morphologies that were clearly nonphysiologic). For analyses of disruptive artifacts (i.e. artifacts occurring during the HIT and likely to affect VOR gain measures by distorting key landmarks), we do not include excessive bounce since this particular artifact does not influence VOR gain interpretation.

We further classified traces as interpretable or uninterpretable with respect to estimating VOR gain. We used morphological rules that considered 'uninterpretable' any trace with a disruptive physiologic eye movement (saccade or nystagmus) or disruptive artifact substantially distorting the bell-shaped curve or obscuring key landmarks required for correct VOR calculation, 


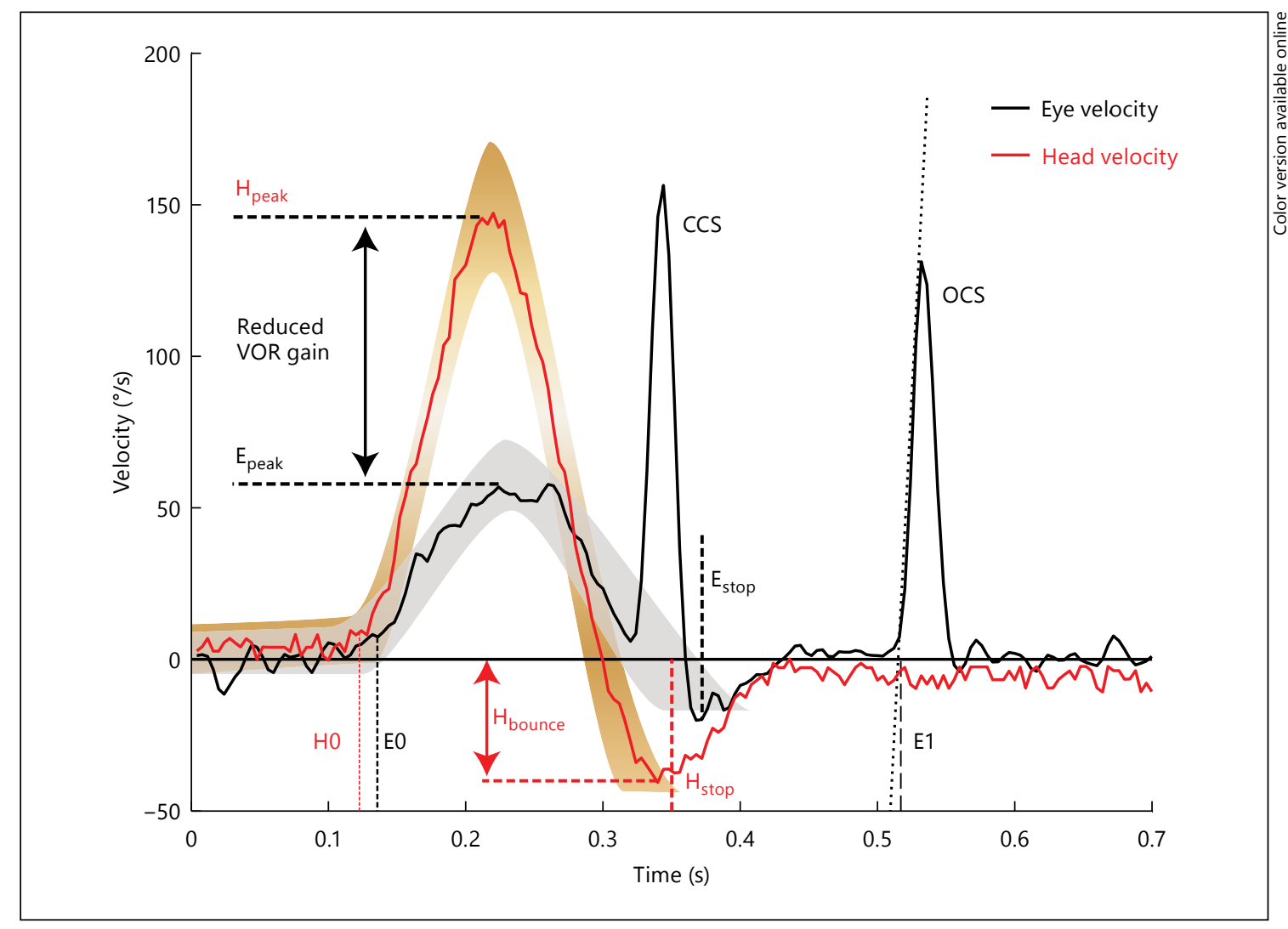

Fig. 1. Physiologic attributes and parameter definitions for a single, typical, abnormal h-HIT trace. Head velocity traces are shown in red, eye velocity in black. Note that eye movements are in the opposite direction to head movements, but are displayed graphically as superimposed to make visual assessment of VOR gain (eye movements relative to head movements) clearer. $\mathrm{H} 0=$ Head velocity onset; $\mathrm{E} 0$ = eye velocity onset; $\mathrm{H}_{\text {peak }}=$ peak head velocity; $\mathrm{E}_{\text {peak }}=$ peak eye velocity; $\mathrm{H}_{\text {bounce }}=$ head velocity crosses baseline with head reversal following deceleration (bounce); $\mathrm{H}_{\text {stop }}=$ head move-

such as the peak VOR eye velocity (online suppl. Appendix B). Neither saccades nor nystagmus were considered artifacts: however, these disruptive eye movements sometimes rendered VOR traces uninterpretable if they occurred prior to the peak head velocity (i.e. near the top of the bell-shaped curve). Fast-phase eye movements (nystagmus or saccades) or artifacts were considered 'nondisruptive' if they did not occur during the slowphase VOR, did not substantially distort VOR morphology (bellshaped curve), or obscure measurement landmarks. For interpretable' HITs, we further classified each as having normal or abnormal gain. Further details about the classification of eye movements and artifacts are described in online supplementary Appendix B.

\section{Statistics}

We used descriptive statistics, reporting the frequency of normal or abnormal HIT graphs and specific artifacts. For interrater agreement, Cohen's kappa was calculated using SPSS software version 17 ment stops; $\mathrm{E}_{\text {stop }}=$ eye movement stops; CCS = covert corrective saccade (during head movement); OCS = overt corrective saccade (after head movement) with dotted line (slope = saccade acceleration) to identify E1 saccade onset; VOR latency = E0 - H0; VOR gain $=$ eye velocity divided by head velocity at a specific time during the HIT (generally $\mathrm{E}_{\text {peak }} / \mathrm{H}_{\text {peak }}$ ) or across a range of times $\left(\mathrm{E}_{\text {times }} / \mathrm{H}_{\text {times }}\right)$ (generally the ratio of the areas under the two curves over the entire HIT duration); saccade latency = E1 - E0.

(SPSS Inc., Chicago, Ill., USA). We considered kappas $<0.4$ poor agreement, 0.4-0.75 fair-to-good agreement, and $>0.75$ excellent agreement [Fleiss, 1981]. A two-sided $\chi^{2}$ test was used to compare proportions of HIT traces with artifacts across diagnostic groups.

\section{Results}

We enrolled 30 acute vestibular syndrome patients and include 26 patients here who had a definitive diagnosis according to structured definitions described above. There were 19 men and 7 women, with a mean age of 60.3 years (range: 31-83, interquartile range: 55-68). Of these, 16 patients had a definite diagnosis of vestibular neuritis, and 10 had MRI-confirmed strokes [7 


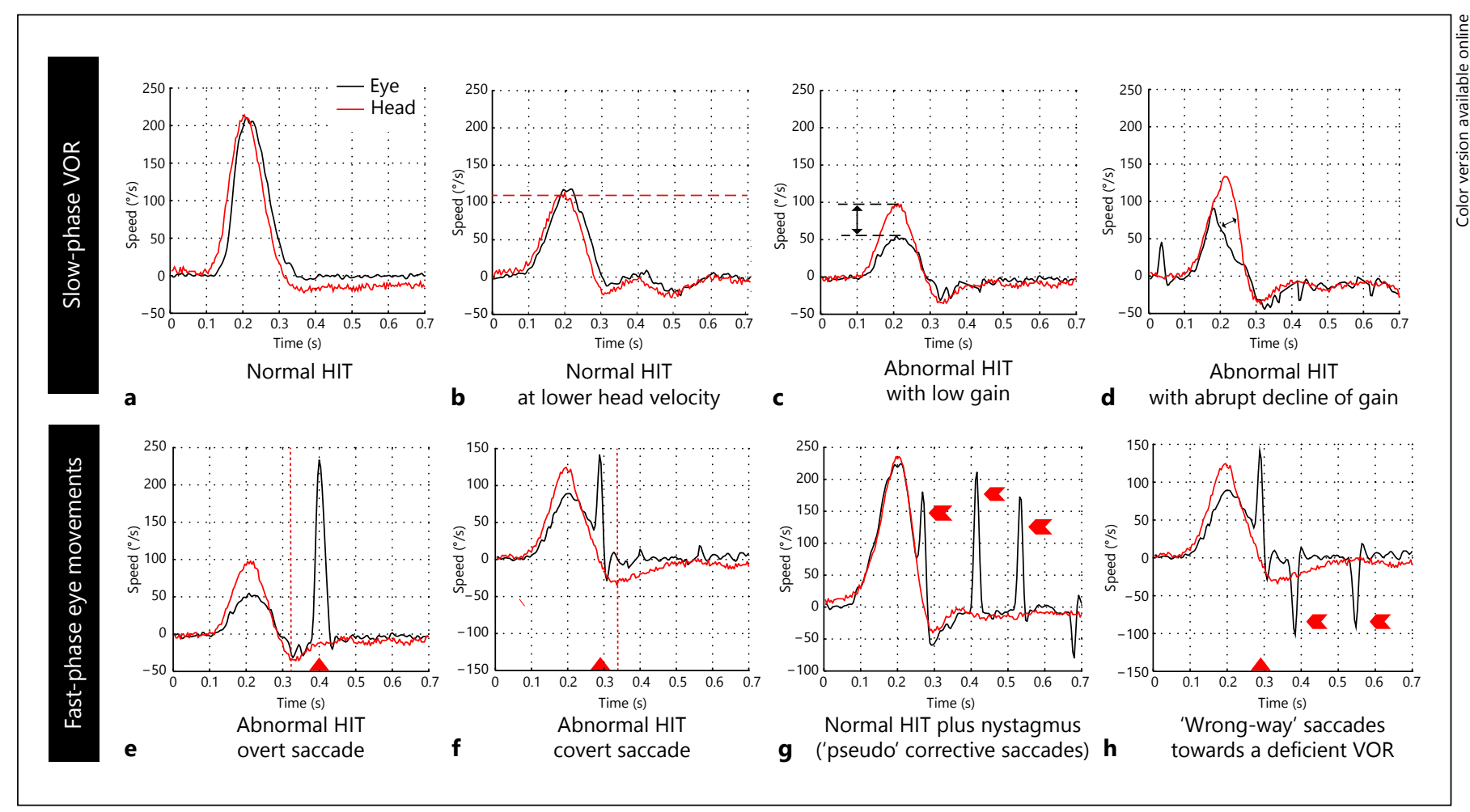

Fig. 2. Exemplar individual HIT traces showing the range of typical normal and abnormal physiologic VOR and saccade findings under various clinical circumstances. Slow-phase VOR gain can be either normal $(\mathbf{a}, \mathbf{b})$ or deficient $(\mathbf{c}, \mathbf{d})$. HITs can be performed through a wide range of head velocities (two examples shown in $\mathbf{a}$ and $\mathbf{b}$ ). The morphology of fast eye movements is the same regardless of underlying physiology, but their location, timing, pattern, and association with normal or abnormal VOR may allow differentiation of saccades (e, $\mathbf{f}, \mathbf{h})$ from nystagmus ( $\mathbf{g}$, possibly $\mathbf{h}$ ). e A typical, overt (late) refixation saccade after a deficient VOR.

posterior inferior cerebellar artery (PICA) territory, 3 anterior inferior cerebellar artery (AICA) territory]. One of the AICA stroke patients had bilateral cerebellar ischemic lesions. All enrolled patients tolerated the hHITs and accomplished the full examination according to the study protocol without complications.

Primary study results are shown in tables $1-3$. We analyzed 1,358 h-HIT graphs from the 26 patients. We found $72 \%$ of HIT traces had abnormal (but pathophysiologically appropriate) disruptive fast eye movements, $44 \%$ had at least one artifact, and $42 \%$ of traces were deemed uninterpretable by visual inspection (despite having been accepted by the device software's internal filtering algorithm). Only $46 \%$ of the traces were both artifact free and without disruptive fast eye movements. Detailed results with interrater agreements are presented in table 1.

Quantifying the VOR with VOG f, h Covert (early) refixation saccades after a deficient VOR response. $\mathbf{g}$ A rhythmic run of nystagmus beats after a normal VOR response (note that the nystagmus beats might be confused for refixation saccades, both covert and overt). $\mathbf{h}$ 'Wrong-way' saccades, probably representing nystagmus towards the VOR deficit. Minor, nondisruptive fixation losses or mini-blinks are present in panels $\mathbf{d}$ (far left of trace) and $\mathbf{g}$ (far right of trace). There is also a small degree of 'noisy baseline' artifact in some of the eye traces, most obvious in panels $\mathbf{d}$ and $\mathbf{f}$.

\section{Slow-Phase VOR}

Of the 1,358 HIT traces, 58\% ( $\mathrm{n}=794 / 1,358,26$ patients) had interpretable VOR results (fig. 2a-d). Of the interpretable results, $61 \%(\mathrm{n}=485 / 794,25$ patients $)$ had clearly normal, $31 \%$ ( $\mathrm{n}=245 / 794,23$ patients) clearly abnormal h-HIT gain, and $8 \%(\mathrm{n}=64 / 794,10$ patients) an abrupt decline of gain (fig. 2d). Mean HIT velocity was $148 \%$ s (SD 39, range: $52-348$ ). Nondisruptive artifacts were present in $22 \%$ of interpretable HITs.

\section{Fast-Phase Eye Movements}

Of all fast phase eye movements, one third could be identified either as a corrective saccade associated with an abnormal HIT or spontaneous nystagmus; however, two thirds could not be readily classified (table 1 ). We found 
Table 1. Morphology of 1,358 h-HIT traces from 26 patients with acute vestibular syndrome

\begin{tabular}{|c|c|c|c|}
\hline \multicolumn{2}{|c|}{ Morphologic attributes of video h-HIT tracing ${ }^{1}$} & $\begin{array}{l}\text { Number of h-HITs, } \\
\mathrm{n}(\%)\end{array}$ & $\begin{array}{l}\mathrm{Kappa}^{2} \\
0.782\end{array}$ \\
\hline Slow-phase VOR & $\begin{array}{l}\text { normal VOR } \\
\text { abnormal VOR without abrupt decline } \\
\text { abnormal VOR with abrupt decline } \\
\text { incorrect morphology (uninterpretable traces) }\end{array}$ & $\begin{array}{l}485(36) \\
245(18) \\
64(5) \\
564(42)\end{array}$ & $\begin{array}{l}0.782 \\
0.548 \\
0.270^{3} \\
0.666\end{array}$ \\
\hline Artifacts $^{5}$ & $\begin{array}{l}\text { one or more artifacts (not counting 'bounce') } \\
\text { no artifacts }\end{array}$ & $\begin{array}{l}599(44) \\
759(56)\end{array}$ & $\begin{array}{l}0.635 \\
0.635\end{array}$ \\
\hline
\end{tabular}

${ }^{1}$ All within-category classifications in this table are mutually exclusive and jointly exhaustive (one and only one per h-HIT). Thus, all percentages in each category sum to $100 \%$.

${ }^{2}$ Cohen's kappa reports the interrater agreement above that expected by chance alone.

${ }^{3}$ Note that in the random $10 \%$ subsample coded by both raters, these events were rare events $(<10$ total). Thus, the kappa values may not represent stable estimates of anticipated agreement in a larger sample.

${ }^{4}$ Includes disruptive artifacts as well as disruptive physiologic fast phase eye movements.

${ }^{5}$ Includes all artifacts except 'bounce' (see Methods). Disruptive fast-phase eye movements (i.e. physiologic nystagmus and saccades) were not considered artifacts.

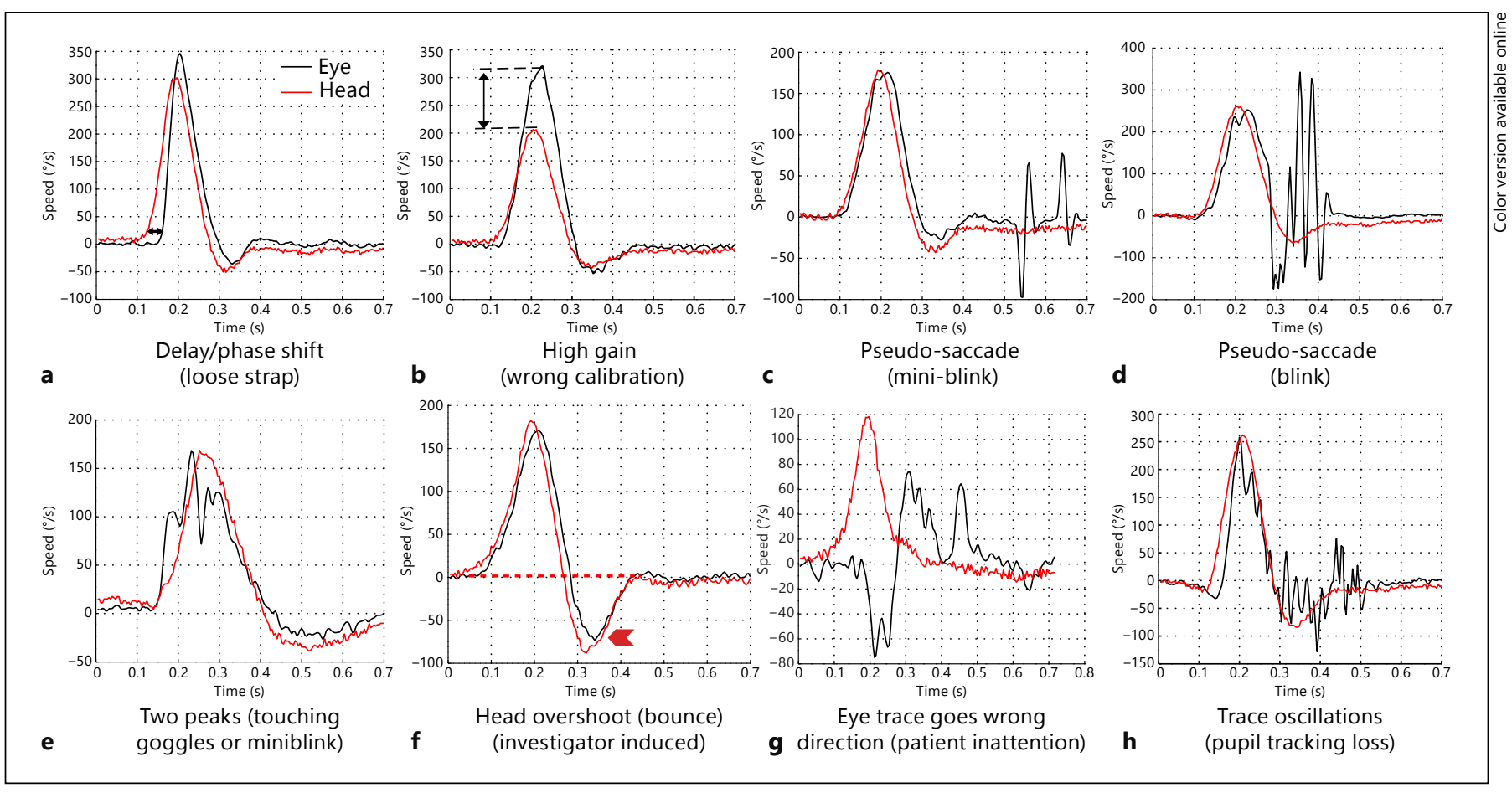

Fig. 3. Exemplar HIT traces demonstrating the range of typical artifacts. a-h depict seven artifacts intentionally generated and reproduced under laboratory conditions in a single normal subject: phase shift (a), inappropriately high gain (b), pseudo-saccades (c, d), multiple VOR peaks (i.e. non-bell-shaped curve) (e), excessive
post-HIT bounce (f), eye moves opposite the expected slow phase VOR direction (i.e. with rather than in opposition to the head) (g), and trace oscillations (noisy baseline) (h). These artifacts of known underlying cause served as the morphologic template for determining the nature of artifacts post hoc in patient traces. 
Table 2. Frequency of artifacts, by type, in 1,358 h-HIT traces from 26 patients with acute vestibular syndrome

\begin{tabular}{llll}
\hline Artifact type & Illustration & $\mathrm{n}$ & $\%$ \\
\hline Any artifact, including bounce $^{1}$ & fig. 1, 3 & 768 & 57 \\
Bounce (head reversal following deceleration) & fig. 3f & 316 & 23 \\
Trace oscillations (suspected pupil tracking loss) & fig. 3h & $211^{2}$ & 16 \\
$\quad$ During head movement & & 150 & 11 \\
$\quad$ After head movement & fig. 3e & 112 & 8 \\
Multiple peaks (suspected touching of the goggles) & Appendix B & 101 & 7 \\
Unclassifiable artifact & fig. 3c, d & $73^{2}$ & 5 \\
Pseudo-saccades (suspected blinks or mini-blinks) & & 45 & 3 \\
$\quad$ After head movement & & 32 & 2 \\
$\quad$ During head movement & fig. 3a & 46 & 3 \\
Phase shift (suspected loose strap) & fig. 3b & 41 & 3 \\
High gain (suspected miscalibration) & fig. 3g & 10 & 1 \\
Wrong-way VOR (suspected patient inattention) & & \\
\hline
\end{tabular}

${ }^{1}$ Bounce is not an artifact, per se, because it accurately reflects the physiology of an incorrectly applied HIT. It should be avoided because it risks distorting ipsilateral test results by stimulating the contralateral VOR.

${ }^{2}$ Some artifacts occurred both during and after a head movement. Thus, the sum of the subtotals ('during' plus 'after') exceeds the total for that class of artifacts.

Table 3. HIT artifacts and uninterpretable traces stratified by diagnostic group

\begin{tabular}{lllllr}
\hline Diagnosis & Peripheral & Peripheral & Central-PICA & Central-AICA & $\mathrm{p}^{1}$ \\
\hline Side & ipsilesional & contralesional & bilateral & bilateral & \\
$\mathrm{HIT}$ & abnormal & normal & normal & normal or abnormal & \\
$\mathrm{n}$ & 456 & 448 & 319 & 135 & $58(43)$ \\
Total artifacts, $\mathrm{n}(\%)$ & $251(55)$ & $176(39)$ & $114(36)$ & $40(30)$ & $<0.001$ \\
Disruptive artifacts ${ }^{2}, \mathrm{n}(\%)$ & $187(41)$ & $144(32)$ & $94(29)$ & $46(34)$ & $<0.002$ \\
Uninterpretable traces $^{3}, \mathrm{n}(\%)$ & $261(57)^{4}$ & $158(35)$ & $99(31)$ & & \\
\hline
\end{tabular}

\footnotetext{
1 Two-sided $\chi^{2}$.

${ }^{2}$ Includes blinks, trace oscillations, phase shift, high gain, or multiple-peak artifacts during head movement, affecting slow-phase VOR and, thus, results interpretation.

${ }^{3}$ Uninterpretable means abnormal slow-phase morphology (regardless of normal or abnormal gain) resulting from a disruptive artifact or disruptive fast phase (nystagmus or corrective saccades).

${ }^{4}$ Patients with vestibular neuritis had more uninterpretable traces than other groups because they had both a greater frequency of disruptive artifacts and a greater frequency of disruptive fast eye movements (e.g. nystagmus).
}

$45 \%$ of all detected refixation saccades were covert (fig. 2f). All 26 acute vestibular syndrome patients had either spontaneous or gaze-evoked nystagmus evident either with or without fixation. However, 5 of 26 patients showed no evidence of nystagmus during the HIT recordings (either due to suppression by visual fixation or eyeposition-dependent nystagmus).

We identified nystagmus in $8 \%(\mathrm{n}=110 / 1,358,15$ patients) as defined in figure $2 \mathrm{~g}$. In $2 \%(\mathrm{n}=28 / 1,358,9 \mathrm{pa}$ - tients) of all HITs, nystagmus rendered VOR gain difficult to interpret. Multiple wrong-way saccades (fig. $2 \mathrm{~h}$ ) were found in $1 \%(n=15 / 1,358,6$ patients $)$.

\section{Artifacts}

The frequency of various artifacts is provided in table 2 . The most frequent artifact identified was bounce (head reversal following deceleration; $23 \%$ ), but the most common artifacts were trace oscillations (16\%) and non-bell-shaped 


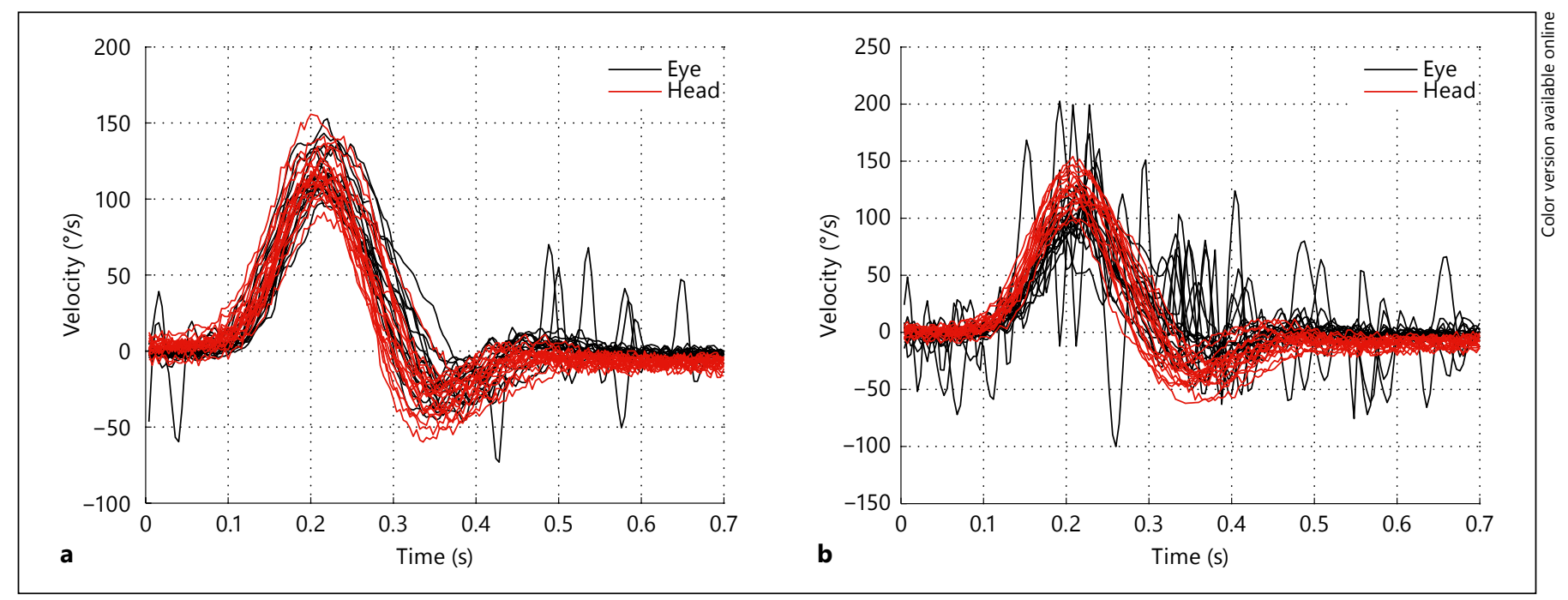

Fig. 4. Back-to-back HIT sessions in a single patient's ear with a normal VOR. Shown are complete, unfiltered device results from two HIT 'runs' from the same ear in the same patient (PICA stroke) obtained during a single visit. Each run shows individual HIT traces temporally superimposed to demonstrate the aggregate result. a Shows a 'clean' run, essentially free of artifacts (without any postprocessing or 'clean up'). The few eye deviations present are either

VOR curves (multiple peaks) (15\%). Unclassifiable artifacts (7\%) and pseudo-saccades from suspected blinks (5\%) were the next most frequent, with other artifacts occurring in only $3 \%$ or fewer of all HITs. The head impulse technique holding the jaw of the patient was associated with more frequent artifacts than when standing behind the patient and holding the top of the head ( $52 \mathrm{vs.} 42 \%, \mathrm{p}=0.005$ ).

We sporadically saw inappropriately high gains $(>1.2)$ in 41 traces from 13 patients; 2 of these patients had systematically higher gains (majority of HIT traces). Figure $3 \mathrm{~b}$ illustrates the higher amplitude of eye velocity after a miscalibration under laboratory conditions.

Artifacts were more frequent in patients with an abnormal VOR (table 3). Patients with PICA strokes (all with bilaterally normal head impulses) had more interpretable traces compared to patients with vestibular neuritis (unilaterally abnormal; 69 vs. $43 \%, \mathrm{p}<0.001$ ). Patients with AICA strokes who displayed variable h-HIT VOR patterns with some normal and others abnormal had intermediate results, but were overall more similar to PICA strokes than to neuritis cases (66\% interpretable).

Figure 4 illustrates the impact of artifacts on composite results, showing two 'runs' of multiple HIT maneuvers in a single patient that happened to include a large number of individual HIT traces without artifacts and with artifacts. small saccades (small fixation losses or nystagmus beats) or nondisruptive blink-related artifacts. b A relatively 'noisy' run, with more substantial artifacts. Here most of the eye deviations appear to be from recording artifacts, which might be further characterized if the HIT morphology were assessed for each individual HIT (rather than aggregated and superimposed).

\section{Discussion}

To our knowledge, this is the first systematic analysis of video HIT morphology and artifacts. We coded and classified 1,358 HITs in 26 acute vestibular patients. We noted eight different types of artifacts. We found a high frequency of h-HIT traces with at least one artifact, particularly in patients with unilateral VOR gain deficits. Some artifacts occurred during the critical phase of head movement, reducing our ability to interpret the VOR gain one third of the time. Since these head impulses were still classified and accepted as a valid head impulse trace by the device algorithm, some of these artifacts might contribute to false gain calculations. We found that spontaneous nystagmus was often difficult to distinguish from corrective saccades in patients with abnormal VOR gains. Coding of completely normal HIT trace morphology had extremely high interrater reliability. Our findings are important because they raise critical technical considerations for obtaining and interpreting VOR gain results from VOG HIT testing, which are used frequently in clinical practice.

\section{Slow-Phase VOR}

The interrater agreement for the interpretation of normal slow phases was excellent (kappa 0.78 ). The ability to train frontline providers and neurology or otolaryngolo-
Mantokoudis et al. 
gy consultants to reliably recognize a normal result is important for acute diagnosis since a bilaterally normal VOR response in a patient with acute vestibular syndrome is highly suggestive of a central nervous system cause (usually stroke) [Newman-Toker et al., 2008; Kattah et al., 2009; Newman-Toker et al., 2013a; NewmanToker et al., 2013b].

Less obvious was the interpretation of an abnormal slow phase with abrupt decline (fig. 2c; kappa 0.27). This pattern is believed by some to represent a video HIT artifact (also called 'bump artifact' [Macdougall et al., 2013]), but it has also been seen using magnetic search coils in older subjects, particularly at high head accelerations, suggesting it may be physiologic [Tian et al., 2001]. In our series, 1 in 5 abnormal h-HITs showed an initial normal slope and an abrupt decline of the slow-phase eye velocity. Theoretically, this could lead to large disparities in VOR measurement across devices that use different VOR gain calculation methods (i.e. relative area under the curve [GN Otometrics, 2013a] versus relative slope [Collewijn and Smeets, 2000]). This may be relevant to vestibular researchers using VOG devices since different devices use different calculation methods. The low kappa value in our study $(0.27)$ suggests that this pattern is difficult to distinguish from other common artifacts based on morphologic characteristics alone. Further study of abrupt decline is warranted, ideally using simultaneous video HIT and search coil recordings.

Occasionally, patients with very brisk nystagmus cannot be assessed by VOG HIT, even with the device set in 'nystagmus' mode. The rapid slow-phase drift can result in failed calibration or make it impossible for the software to calculate initial eye position during the HIT. In all of our studies to date, however, we have identified only one such patient. This may be because primary position nystagmus, if present, is often suppressed by visual fixation in acute vestibular patients. Alternatively, it could be because nystagmus is briefly suppressed as a result of the head impulse itself (e.g. fig. $2 \mathrm{~g}$ ). In those who can be measured, it remains unknown to what extent spontaneous nystagmus might influence VOR gain measures.

\section{Fast-Phase Eye Movements}

Fast-phase eye movements typically occur with a deficient VOR because a corrective refixation saccade makes up for the VOR deficiency. However, they also occur in jerk nystagmus (i.e. quick phases). One of the clinical challenges facing providers interpreting the results of the qualitative clinical HIT in an acute vestibular patient is differentiating a refixation saccade (indicating a VOR de- ficiency) from a beat of spontaneous nystagmus. Typically, either of these fast eye movements is directed towards the contralesional side in an acute unilateral peripheral vestibulopathy. Expert clinicians use subtle visual clues to identify the refixation saccade as distinct from the other nystagmus beats, such as being slightly out of phase or of a slightly larger amplitude.

A VOG device has the ability to visualize fast eye movements and could potentially help clinicians to identify refixation saccades in patients with brisk spontaneous nystagmus, which might otherwise be difficult. In roughly one third of the HIT traces with fast eye movements, we distinguished refixation saccades from nystagmus with fair-to-excellent interrater agreement (kappa 0.53, 0.91) by focusing on rhythmic runs of three or more nystagmus beats (fig. 2g). We also noted 'wrong-way' saccades (fig. $2 \mathrm{~h}$ ) where possible nystagmus beats were directed towards a VOR deficit (opposite a refixation saccade). Unfortunately, corrective saccades and nystagmus fast phases have very similar physiologic properties [Garbutt et al., 2001], and subtle differences in the amplitude of the fast phase cannot be reliably gauged by peak fast eye movement velocity (i.e. height of the saccade 'spike'). This was reflected in the large fraction of h-HIT traces where fast phases could not be definitively classified as one or the other. These results suggest that although the presence of refixations on HIT traces in patients without nystagmus (e.g. chronic unilateral or bilateral vestibulopathy) may be helpful diagnostically [MacDougall et al., 2009; Weber et al., 2009; Macdougall et al., 2013], less should be inferred from the fast eye movements when spontaneous nystagmus is present. Fortunately, unlike with the qualitative clinical HIT, the presence of a corrective eye movement is not critical to identifying the VOR deficit using a VOG device. Instead, the directly measured VOR gain deficit appears to be sufficient [Newman-Toker et al., 2013b]. Given our results above regarding slow phases, our results suggest that the primary focus should generally be on VOR morphology with only secondary consideration given to the presence or absence of saccades, unless there is known to be no spontaneous nystagmus.

'Wrong-way' saccades (fig. 2h) were an interesting finding which occurred infrequently (1\% of traces). It is unclear whether these represented nystagmus beats directed towards a deficient VOR ('wrong-way' nystagmus), refixations following overshoot (either due to bounce or a miscalibrated refixation saccade), or extraneous saccades (e.g. loss of fixation; see online suppl. Appendix B for further discussion). 


\section{HIT Trace Artifacts}

Artifacts were surprisingly common, with one or more artifacts seen in nearly half of the collected impulses and disruptive artifacts seen in more than a third. Many of the artifacts were probably induced by the examiners themselves (e.g. 'bounce' in delivering impulse, touching goggles), despite their extensive training and experience. Technique also matters - holding the top of the head instead of the jaw has a slightly lower risk for creating iatrogenic artifacts, although this difference could also have been attributable to specific examiner skill since each site consistently applied only one technique. Interestingly, artifacts occurred 10-20\% more often (absolute increase) in patients with abnormal h-HITs, a difference that was highly statistically significant (table 3 ). Whether this reflects misclassification (i.e. physiologic disruptive eye movements erroneously called artifacts) or a true effect of the impaired VOR (e.g. due to greater risk of pupil tracking loss with more refixation saccades) merits additional study.

The most common 'artifact' (technique error) was head 'bounce' (table 2). This artifact results from the examiner inadvertently rotating the head back in the opposite direction (rather than coming to a full stop) at the end of the impulse, following deceleration. Bounce was generally minor, and only adversely affected trace interpretation if severe. In our experience, bounce only affects data quality with novice examiners (first hour of training).

The second most common artifact was noisy eye traces with multiple VOR peaks (i.e. non-bell-shaped morphology; fig. 3e). This trace morphology could be easily reproduced under laboratory conditions by touching the goggles directly or indirectly (e.g. by touching the strap). However, multiple peaks could also be created by a miniblink without pupil tracking loss or by nystagmus with a quick phase in the opposite direction. Regardless of the cause, this artifact was the most common reason for an uninterpretable trace.

Another major source of artifacts was pupil tracking loss resulting in oscillations on the trace or even loss of the recording trace (fig. $3 \mathrm{~h}$ ). This was the second most common cause of an uninterpretable trace. Pupil tracking can be affected by environmental lighting conditions via miosis or reflected light off the cornea, both of which reduce pupil-tracking efficiency. Optimal lighting conditions appeared to be soft, even relatively low light, although recordings were generally robust to the typical emergency department or clinic room lighting. Pupil tracking can also be affected by the position of the mirroring infrared LEDs [Eggert, 2007], narrow palpebral fissures (eyelid ptosis, brow ptosis, congenital/racial, etc.), or even lightreflecting makeup (e.g. eyelash mascara or eyelid liner). Independent of the cause, we found that pupil tracking could usually be improved by adjusting software settings (e.g. pupil detection thresholds). The algorithm of our VOG system was efficient and rejected $98 \%$ of h-HITs after detection of a pupil tracking loss.

Other artifact types were uncommon in our series $(\leq 5 \%)$. Goggle slippage during head acceleration occurred if the goggles were not attached firmly enough. This problem can cause an artifact with an undershooting of the eye velocity curve at the beginning of a head movement [Bartl et al., 2009]. In addition, the eye graph might lead the head graph (phase shift; fig. 3a) or vice versa. Latency between the start of a head movement and the compensatory eye movement has a physiological range of 7-15 ms, which should be taken into account [Leigh and Zee, 2006]. Video goggle slippage due to a high mass inertia [Bartl et al., 2009; Weber et al., 2009] has been described previously and a model for slippage compensation proposed [Bartl et al., 2009]. A similar phenomenon can be seen with the search coil technique with a coil slippage on the conjunctiva [Jorns-Haderli et al., 2007]. In our series, only $3 \%$ of HITs showed a significant phase lag $(>20$ $\mathrm{ms}$ ) between eye and head trace due to goggle slippage.

\section{VOR Gain Distortions due to Artifacts}

A complete analysis of the effect of artifacts on VOR gain and diagnostic classification is beyond the scope of this article and will be presented elsewhere. We note, however, that a particular error, incorrect calibration, consistently resulted in false gain elevations. We were able to reproduce traces with gains above 1.2 in normal subjects under laboratory conditions by performing repeated HITs with an inaccurate calibration (fig. 3b). This might occur naturally in patient who was inattentive during calibration. Some of our patients were drowsy or distracted and did not look accurately at the projected laser dot; others had brisk nystagmus that probably affected the quality of fixation and, hence, calibration. In some cases, recalibration was required to obtain valid results during our testing protocols.

Apparently physiologic high gains (at least up to 1.2) can be observed for HITs [MacDougall et al., 2009; Agrawal et al., 2014] with fixation distance near rather than far or in patients with spectacle corrections [Cannon et al., 1985]. They can also occur in patients with cerebellar disease due to disinhibition of the vestibular nuclei, although this has only been shown in patients with bilateral chronic lesions, rather than those with acute unilat-
48

Audiol Neurotol 2015;20:39-50 DOI: $10.1159 / 000362780$
Mantokoudis et al. 
eral ones [Walker and Zee, 2005]. We found 2 out of our 26 patients with mean VOR gains above 1.2. One had a stroke in the left lateral medulla and bilateral elevated gain values; the other had a left vestibular neuritis with elevated gains on the contralesional side. Both showed major artifacts on most of the traces, rendering most of the measurements obtained uninterpretable. We concluded the high mean gains were likely artifactual.

Although these results might seem concerning for VOG interpretive validity, additional recent analyses suggest that artifacts primarily introduce random noise, and overall diagnostic classification is not meaningfully affected [Mantokoudis, unpubl.]. The high frequency of artifacts does decrease precision, so relying on fewer than 10 unfiltered HITs for mean VOR gain measures is not advisable. Manual filtering of artifacts is also recommended.

\section{Limitations}

We did not validate our impulse findings using scleral search coil recordings. Artifacts were determined based on morphology alone, so we cannot be sure all were artifacts. Our methods likely overstate the frequency of artifacts somewhat because some of the traces might have been physiologic rather than artifactual, per se. Non-realtime analysis of eye traces did not allow strong inferences to be drawn about the underlying causes for artifacts in each specific case. Coding reliability was low for some types of artifacts. Exam technique differences might have influenced results. We tested only one VOG head impulse device, and it remains unknown whether our results generalize to other similar recording systems. Finally, there was no healthy, asymptomatic control group for comparison of artifact frequency.

\section{Potential Implications}

Since video HIT has gained popularity among ENT physicians and neurologists, knowledge about the correct interpretation of quantitative h-HITs is essential. Our results indicate that use of video goggles in patients with acute vertigo should currently be limited to providers familiar with the range of possible artifacts and their diagnostic implications. Some artifacts from blinks or inattention are probably unavoidable, while others are technique dependent (e.g. bounce). Familiarity with a coding manual (fig. 1-3; online suppl. Appendix B) might prove valuable to practitioners adopting this technology. Our findings also suggest it may be easier to train physicians or technicians to reliably identify truly normal traces than to differentiate abnormal traces from artifacts. The ability for frontline providers to do so might offer the possibility to speed identification of acute stroke in acute vestibular syndrome patients, including in the emergency department or even prehospital care setting [Newman-Toker et al., 2013b]. In most cases it would also help correctly select the most appropriate clinical consultant to further evaluate and treat a patient's acute dizziness or vertigo-stroke: neurologist for bilaterally normal VOR results, or a vestibular otologist to distinguish artifacts from true unilateral or bilateral abnormal VOR results.

\section{Conclusions}

As video h-HIT testing appears to be associated with a high frequency of artifacts, training in interpretation is essential. Awareness of the most common artifacts can help practitioners avoid creating them (for those which are iatrogenic) or misinterpreting the results when they occur. Further study is essential to determine the clinical diagnostic implications of artifacts frequently encountered during video HIT testing as the use of this technique increases.

\section{Acknowledgements}

This study was supported by the Swiss National Science Foundation (PBBEP2 136573).

\section{Disclosure Statement}

The authors report no conflicts of interest.

References
E, Zee D, Carey J: Evaluation of quantitative
head impulse testing using search coils vs.
video-oculography in older individuals. Otol
Neurotol 2014;35:283-288.
Bartl K, Lehnen N, Kohlbecher S, Schneider E:
Head impulse testing using video-oculogra-
phy. Ann NY Acad Sci 2009;1164:331-333.
Blödow A, Helbig R, Bloching M, Walther LE:
Isolated functional loss of the lateral semicir-
cular canal in vestibular neuritis (in German).
HNO 2013;61:46-51.


Cannon SC, Leigh RJ, Zee DS, Abel LA: The effect of the rotational magnification of corrective spectacles on the quantitative evaluation of the VOR. Acta Otolaryngol 1985;100:81-88.

Collewijn H, Smeets JB: Early components of the human vestibulo-ocular response to head rotation: latency and gain. J Neurophysiol 2000; 84:376-389.

Curthoys IS: The interpretation of clinical tests of peripheral vestibular function. Laryngoscope 2012;122:1342-1352.

Eggert T: Eye movement recordings: methods. Dev Ophthalmol 2007;40:15-34.

-Fleiss JL: Statistical Methods for Rates and Proportions. Hoboken, Wiley, 1981.

Garbutt S, Harwood MR, Harris CM: Comparison of the main sequence of reflexive saccades and the quick phases of optokinetic nystagmus. Br J Ophthalmol 2001;85:1477-1483.

GN Otometrics: ICS Impulse. http://www.icsimpulse.com/.

GN Otometrics: ICS Impulse FAQ. 2013a. http:// www.otometrics.com/ /media/DownloadLibrary/Otometrics/PDFs/ICS\%20Impulse/ 7-26-8801-EN_04_STD.pdf.

GN Otometrics: Video head impulse test - datasheet. 2013b http://www.otometrics.com/ / media/DownloadLibrary/Otometrics/PDFs/ ICS\%20Impulse/7-26-9899-EN_02_STD. pdf.

- Halmagyi GM, Aw ST, Cremer PD, Curthoys IS, Todd MJ: Impulsive testing of individual semicircular canal function. Ann NY Acad Sci 2001;942:192-200.

Halmagyi GM, Curthoys IS: A clinical sign of canal paresis. Arch Neurol 1988;45:737-739.

-Janky KL, Zuniga MG, Carey JP, Schubert M: Balance dysfunction and recovery after surgery for superior canal dehiscence syndrome. Arch OtolaryngolHead Neck Surg 2012;138:723730.

-Jorns-Haderli M, Straumann D, Palla A: Accuracy of the bedside head impulse test in detecting vestibular hypofunction. J Neurol Neurosurg Psychiatry 2007;78:1113-1118.
Kattah JC, Talkad AV, Wang DZ, Hsieh YH, Newman-Toker DE: HINTS to diagnose stroke in the acute vestibular syndrome: three-step bedside oculomotor examination more sensitive than early MRI diffusion-weighted imaging. Stroke 2009;40:3504-3510.

Kim JS, Kim HJ: Inferior vestibular neuritis. J Neurol 2012;259:1553-1560.

Lehnen N, Aw ST, Todd MJ, Halmagyi GM: Head impulse test reveals residual semicircular $\mathrm{ca}$ nal function after vestibular neurectomy. Neurology 2004;62:2294-2296.

Leigh RJ, Zee DS: The vestibular-optokinetic system; in Leigh RJ, Zee DS, (eds): The Neurology of Eye Movements. New York, Oxford University Press, 2006, pp 20-107.

-Macdougall HG, McGarvie LA, Halmagyi GM, Curthoys IS, Weber KP: The video Head Impulse Test (vHIT) detects vertical semicircular canal dysfunction. PloS One 2013;8: e61488.

MacDougall HG, Weber KP, McGarvie LA, Halmagyi GM, Curthoys IS: The video head impulse test: diagnostic accuracy in peripheral vestibulopathy. Neurology 2009;73:11341141.

Manzari L, Burgess AM, MacDougall HG, Bradshaw AP, Curthoys IS: Rapid fluctuations in dynamic semicircular canal function in early Meniere's disease. Eur Arch Otorhinolaryngol 2011;268:637-639.

-Manzari L, MacDougall HG, Burgess AM, Curthoys IS: New, fast, clinical vestibular tests identify whether a vertigo attack is due to early Meniere's disease or vestibular neuritis. Laryngoscope 2013;123:507-511.

Newman-Toker DE: Symptoms and signs of neuro-otologic disorders. Continuum 2012;18: 1016-1040.

- Newman-Toker DE, Kattah JC, Alvernia JE, Wang DZ: Normal head impulse test differentiates acute cerebellar strokes from vestibular neuritis. Neurology 2008;70:2378-2385.

Newman-Toker DE, Kerber KA, Hsieh YH, Pula JH, Omron R, Saber Tehrani AS, Mantokoudis G, Hanley DF, Zee DS, Kattah JC: HINTS outperforms ABCD2 to screen for stroke in acute continuous vertigo and dizziness. Acad Emerg Med 2013a;20:986-996.
Newman-Toker DE, Saber Tehrani AS, Mantokoudis G, Pula JH, Guede CI, Kerber KA, Blitz A, Ying SH, Hsieh YH, Rothman RE, Hanley DF, Zee DS, Kattah JC: Quantitative videooculography to help diagnose stroke in acute vertigo and dizziness: toward an ECG for the eyes. Stroke 2013b;44:1158-1161.

Park HJ, Migliaccio AA, Della Santina CC, Minor LB, Carey JP: Search-coil head-thrust and caloric tests in Meniere's disease. Acta Otolaryngol 2005; 125:852-857.

Petersen JA, Straumann D, Weber KP: Clinical diagnosis of bilateral vestibular loss: three simple bedside tests. Ther Adv Neurol Disord 2013;6:41-45.

Robinson DA: A method of measuring eye movement using a scleral search coil in a magnetic field. IEEE Trans Biomed Eng 1963;10:137145.

-Strupp M, Brandt T: Peripheral vestibular disorders. Curr Opin Neurol 2013;26:81-89.

Tarnutzer AA, Berkowitz AL, Robinson KA, Hsieh YH, Newman-Toker DE: Does my dizzy patient have a stroke? A systematic review of bedside diagnosis in acute vestibular syndrome. CMAJ 2011;183:E571-E592.

Tian JR, Shubayev I, Baloh RW, Demer JL: Impairments in the initial horizontal vestibuloocular reflex of older humans. Exp Brain Res 2001;137:309-322.

Walker MF, Zee DS: Asymmetry of the pitch vestibulo-ocular reflex in patients with cerebellar disease. Ann NY Acad Sci 2005;1039:349358.

Weber KP, Aw ST, Todd MJ, McGarvie LA, Curthoys IS, Halmagyi GM: Head impulse test in unilateral vestibular loss: vestibulo-ocular reflex and catch-up saccades. Neurology 2008; 70:454-463.

Weber KP, MacDougall HG, Halmagyi GM, Curthoys IS: Impulsive testing of semicircularcanal function using video-oculography. Ann NY Acad Sci 2009;1164:486-491.

Young LR, Sheena D: Survey of eye movement recording methods. Behav Res Methods 1975;7: 397-429. 